\title{
La désorption nucléée en génie chimique
}

\author{
Gabriel Wild et Noël Midoux \\ Laboratoire des sciences du génie chimique - Nancy
}

La désorption de gaz à partir de solutions sursaturées est un phénomène qui intervient très fréquemment dans les industries chimique, biochimique et alimentaire: détente de solutions saturées, fermentations, réactions entre composants liquides conduisant à des produits volatiles et peu solubles, électrolyses, réactions parasites lors d'électro-synthèse. La désorption peut avoir lieu de manière diffusionnelle (c'est-à-dire sans formation de bulles), ou de manière nucléée. Si le premier type a été étudié par plusieurs auteurs, le second n'a fait l'objet que de quelques rares travaux soit purement empiriques et non généralisables, soit théoriques et difficilement applicables. Les auteurs présentent l'état des connaissances dans ce domaine et indiquent quelques voies de recherche d'intérêt.

\section{Introduction}

La désorption d'un gaz à partir d'une solution sursaturée est un phénomène extrêmement courant dans le domaine des génies chimique et biochimique. On distingue deux sortes de désorption: la désorption sans formation de bulles (désorption "tranquille ") et la désorption nucléée.

Nous présenterons dans un premier temps un certain nombre de cas, dans lesquels la désorption (en général nucléée) joue un rôle important et dans lesquels la mauvaise connaissance de ce phénomène empêche un dimensionnement fiable de réacteurs. Dans un deuxième temps, nous présenterons très schématiquement les différentes approches de la désorption nucléée proposées par la physico-chimie et verrons en quelle mesure les corrélations obtenues sont applicables dans la réalité industrielle; enfin, nous indiquerons quelles approches de type "génie des procédés " ont été utilisées à ce jour pour pallier la méconnaissance fondamentale du processus.

\section{Importance industrielle de la désorption nucléée}

En première approche, deux types de causes peuvent provoquer la sursaturation d'un liquide par un gaz et entraîner un risque de désorption :

- réaction chimique ou biologique avec dégagement d'une espèce volatile,

- déséquilibre thermodynamique physique induit (pression, température).

\subsection{Désorption par sursaturation physique}

\subsubsection{Dimensionnement des soupapes de sécurité et des disques de rupture [9]}

Il est quelquefois nécessaire de diminuer brusquement la pression au-dessus d'un réservoir de liquide stocké sous pression. Les dimensions nécessaires des orifices de sécurité dépendent de la vitesse de désorption du gaz dissous, la désorption ayant en général lieu par désorption nucléée.

\section{Nucleated desorption in chemical engineering.}

Gas desorption from supersaturated solutions is a phenomenon which occurs very frequently in the chemical, biochemical and food industries: expansion of saturated solutions, fermentations, reactions between liquid elements resulting in volatile and slightly soluble products, electrolyses and parasitic reactions during electrosynthesis. Desorption may occur diffusionally (i.e. without bubbles forming) or nucleated. If the first type has been examined by several authors, the second has only been rarely considered, either purely in an empirical way and which cannot be generalized, or in a theoretically and difficult to apply manner. The authors are submitting what they know of this field and indicate several channels of fruitful research. 


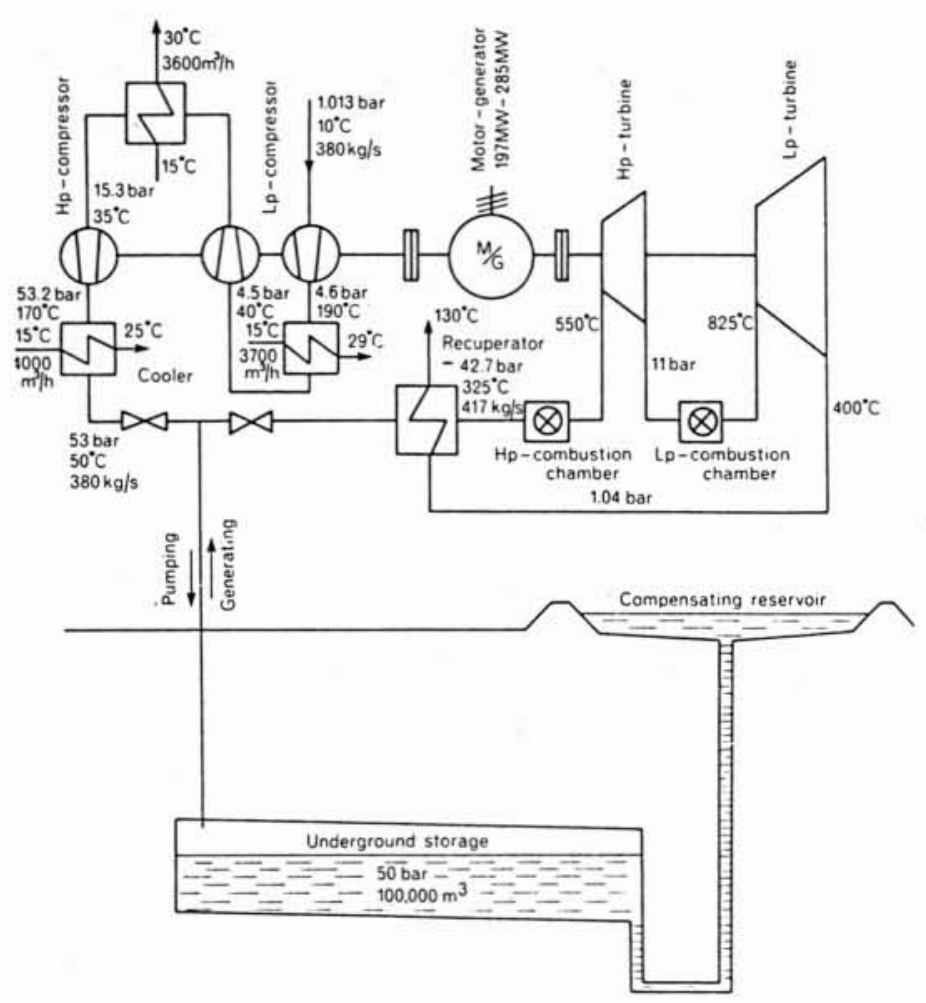

1. Stockage d'énergie dans les sites souterrains: schéma de l'installation de Vianden (RFA) [34]

\subsubsection{Désorption par diminution de la pression statique}

Nous regroupons sous ce titre plusieurs problèmes très divers :

- le mal du plongeur: à la remontée, la désorption nucléée d'azote dissous dans le sang d'un plongeur peut conduire à des accidents graves; c'est la raison des paliers de décompression;

- dans les procédés de stockage d'énergie par compression d'air dans des cavernes souterraines [4, 34], l'énergie est stockée durant les heures creuses par pompage d'air dans une caverne étanche souterraine située à une profondeur d'environ $500 \mathrm{~m}$ et contenant de l'eau et reliée par une jambe manométrique à un étang ou un lac en surface. Aux heures de forte demande, on récupère l'énergie à l'aide de la même pompe fonctionnant en turbine. Si la durée de stockage est longue, l'eau qui est au contact de l'air sous pression se sature. On risque alors que, dans la jambe manométrique, l'air dissous désorbe en quantité telle qu'il se produit un effet de gazosiphon vidant toute l'eau et une bonne partie de l'air comprimé stocké de la caverne. La figure 1 décrit une telle installation réalisée à Vianden (RFA) par la Société électrique de l'Our (Luxembourg) et par la société Brown Boveri [34];

- la cavitation en conduite lors du transport d'un liquide contenant un gaz dissous;

- l'ozonification d'eaux usées à l'aide de jets d'eau saturée sous pression. Le but de cette étude [33] était de se servir de la solubilité assez important de l'ozone en saturant sous pression un assez petit débit d'eau et d'ozonifier l'eau en mélangeant directement cette eau saturée sous pression avec l'eau à traiter par l'intermédiaire de buses d'injection, si possible sans réapparition d'une phase gazeuse. Une étude menée avec un gaz modèle, le $\mathrm{CO}_{2}$ a montré que, en règle générale, il est impossible d'éviter totalement la désorption lors de l'injection.

\subsection{Désorption par sursaturation chimique ou biochimique}

\subsubsection{Procédés biotechnologiques}

Dans les fermentations aérobies, les microorganismes produisent du dioxyde de carbone; pour la commande de ces fermenteurs, il est important d'être en mesure de prédire l'importance du taux de désorption nucléée à une sursaturation donnée. C'est le cas pour la fermentation de la bière [20].

Lors du traitement biologique anaérobie d'eaux usées, la désorption ou non de dioxyde de carbone peut avoir une influence sur le $\mathrm{pH}$ du milieu et, partant, sur le métabolisme des micro-organismes présents.

Des photobioréacteurs sont en essai depuis un certain nombre d'années pour tenter d'étendre à l'échelle industrielle la culture de microalgues, dans lesquelles du $\mathrm{CO}_{2}$ est transformé en matière organique et en oxygène sous l'action du rayonnement solaire. La solubilité de l'oxygène étant beaucoup plus faible que celle du $\mathrm{CO}_{2}$, le bon fonctionnement d'une telle installation peut être perturbé, s'il y a désorption de bulles d'oxygène au contact des microalgues [17].

\subsubsection{Procédés de l'industrie chimique}

La désorption nucléée est particulièrement étudiée dans le cadre de l'électrochimie. Lors de l'électrolyse de l'eau par exemple, on sait que le dégagement d'hydrogène et d'oxygène conduit à des sursaturations locales considérables, mais on ne sait toujours pas si les bulles se "détachent " des électrodes ou se forment par désorption nucléée classique en phase liquide. De même, dans certaines synthèses électrochimiques, des réactions parasites produisent des gaz (p. ex. par électrolyse du solvant). L'apparition ou non de bulles au voisinage de l'électrode peut profondément modifier le transfert de matière liquide-solide et la sélectivité du procédé.

De très nombreux procédés d'épuration des gaz $\left(\mathrm{CO}_{2}\right.$, $\left.\mathrm{H}_{2} \mathrm{~S}, \mathrm{SO}_{2} \ldots\right)$ par lavage par un solvant sélectif [25-27], mettent en œuvre une absorption avec réaction chimique réversible à " haute " température; ce choix permet de régénérer le solvant en libérant le gaz. Ainsi, on met fréquemment en œuvre l'absorption de $\mathrm{CO}_{2}$ dans des solutions d'alcanolamines ou de carbonates à température ambiante, les carbamates ou hydrogénocarbonates étant décomposés à chaud (environ $80^{\circ} \mathrm{C}$ ) avec désorption de $\mathrm{CO}_{2}[15,18,19,23,24]$. Remarquons cependant que cette régénération (stripping) est en général traitée comme une désorption non nucléée.

Dans certains cas, il peut y avoir désorption de gaz créé par le procédé d'épuration lui-même. Un exemple $[21,22]$ est la purification d'air contenant $\mathrm{NO}_{\mathrm{x}}$ et $\mathrm{SO}_{2}$. 
Une des étapes chimiques intervenant dans le procédé est la réaction des ions nitrite avec l'acide sulfamique, réaction qui produit de l'azote, qui désorbe.

Lors de l'attaque de solides par voie acide : que le but soit de dissoudre un solide [1] ou de neutraliser un effluent acide à l'aide de calcaire [30], la vitesse de désorption du gaz formé peut être déterminante pour l'ensemble du procédé.

Le dégagement des produits gazeux limite les performances des procédés de liquéfaction de charbon [2].

Polymérisations avec production de gaz (polymères expansés, éponges, désorption d'eau limitant certaines réactions en milieu polymérique fondu...)

\subsubsection{Autres branches de lindustrie}

Dans l'affinage du verre, la décomposition de certains ingrédients produit des bulles de petite taille que l'on est obligé d'éliminer en injectant des bulles de grande taille qui les captent dans leur sillage.

Dans la désoxygénation de fonte d'acier sous vide, le carbone réagit avec l'oxygène dissous pour former de l'oxyde de carbone qui désorbe [16].

\section{Travaux théoriques et vérification expé- rimentale}

Ces travaux portent sur la nucléation homogène, due à la formation des bulles dans un liquide "propre ", ainsi que sur la nucléation hétérogène qui fait intervenir des germes (microbulles ou particules solides) ou des sites de nucléation (sur des parois solides macroscopiques).

\subsection{La nucléation homogène}

La théorie classique de la nucléation homogène due initialement à Volmer [29] est résumée par Blander [3] dans un article de synthèse qui concerne d'ailleurs surtout l'autovaporisation des liquides et plus précisément le régime "explosif". Dans le cadre de cette théorie, toute association de $i$ molécules ( $i=1$ dans le cas des gaz parfaits) constitue potentiellement un germe de nucléation. Si la microbulle ainsi formée atteint une taille critique, elle ne peut que croitre. Dans le cas de la désorption nucléée d'un gaz peu soluble à partir d'une solution sursaturée, les molécules doivent diffuser vers le germe de nucléation et la vitesse de nucléation prévue par la théorie est du type :

$J \approx D\left(C_{G}-C_{L}\right)\left(\frac{k T}{\sigma}\right)^{1 / 2} \exp \left[-\frac{1,21.10^{24} \sigma^{3}}{T\left(P_{G}-P_{L}\right)^{2}}\right]$

où $P_{G}$ est la pression de saturation et $P_{L}$ la pression après dépressurisation. On constate que la vitesse de nucléation $J$ est une fonction "raide " de la pression, fait qui se traduit par l'existence d'un seuil critique de sursaturation (ou de dépression) en-dessous duquel la nucléation homogène reste négligeable. Par ailleurs, la vitesse de nucléation prédite par cette relation dépend énormément de la tension superficielle $\sigma$.
Nous n'insisterons pas sur cette théorie, infirmée par les quelques informations expérimentales dont on dispose. Finkelstein et Tamir [8] sont, à notre connaissance, les premiers à avoir mis en évidence expérimentalement l'existence de la désorption nucléée homogène, en saturant sous très haute pression $(100 \mathrm{MPa})$ de l'eau ou des solutions aqueuses avec un certain nombre de gaz (He, $\mathrm{Ne}, \mathrm{N}_{2}, \mathrm{Ar}$ ). A ces pressions, la nucléation hétérogène ne joue plus aucun rôle, les microbulles présentes soit au sein du liquide soit dans les anfractuosités des parois étant dissoutes. Les résultats obtenus par ces auteurs confirment ceux obtenus à des pression plus faibles par Hemmingsen dans des tubes capillaires [10] ou dans des cellules de protozoaires [11].

La dépression nécessaire $\Delta P_{N}$ pour obtenir la nucléation ne dépend pas (ou très peu) de la tension superficielle, de la densité de la solution et de sa force ionique. C'est une valeur caractéristique du gaz utilisé, indépendante du niveau de pression de départ.

Afin d'expliquer leurs résultats, Finkelstein et Tamir ont élaboré un modèle fondé sur la thermodynamique des processus irréversibles. Le solvant est considéré contenir des groupes de molécules (4 à 10) de gaz dissous qui se forment et se détruisent en régime permanent. En-dessous de la dépression critique, le phénomène de diffusion moléculaire susceptible d'alimenter ces microbulles est trop lent pour conduire à la nucléation. Au-delà de la dépression critique, l'alimentation de ces sites s'effectue aussi par convection forcée dans des canaux dont le diamètre est identique au diamètre moléculaire $d_{m}$. Egalant les fonctions de dissipation des processus diffusionnel (régi par la loi de Fick) et convectif (régi par la loi de Poiseuille), explicitant le coefficient de diffusion à partir de la loi de Wilke et Chang, ces auteurs aboutissent à :

$$
\frac{\Delta P_{N}}{P_{c}}=\operatorname{Cte} \frac{V_{G D} V_{\dot{e} \bar{b}}^{0.6}}{d_{m}^{4}}\left(\frac{T}{T_{c}}\right)
$$

Il est évidemment difficile de juger de la généralité à l'heure actuelle d'un tel modèle qui comporte un certain nombre d'hypothèses uniquement justifiées par un accord très correct avec l'expérience. Dans l'eau, pour les différents gaz expérimentés, les auteurs ont établi la corrélation empirique :

$$
\frac{\Delta P_{N}}{P_{c}}=1,3\left(\frac{T}{T_{c}}\right)^{1,2}
$$

La figure 2 (page suiv.) illustre la qualité de cette corrélation empirique.

\subsection{La nucléation hétérogène}

Industriellement la nucléation est surtout hétérogène, soit à partir d'agrégats moléculaires en solution, soit surtout à partir de petits domaines gazeux retenus par des sites aux parois. Ces cavités de rayon $R_{0}$ sont actives pour la nucléation si la dépression $\left(P_{G}-P_{L}\right)$ est supérieure à celle prévue par la loi de Laplace $[6,7,31]$ soit, en admettant l'applicabilité de la loi de Henry:

$$
R_{0} \geqslant \frac{2 \sigma}{P_{G}-P_{L}}=\frac{2 \sigma}{\mathrm{He} \cdot\left(C_{G}-C_{L}\right)}
$$




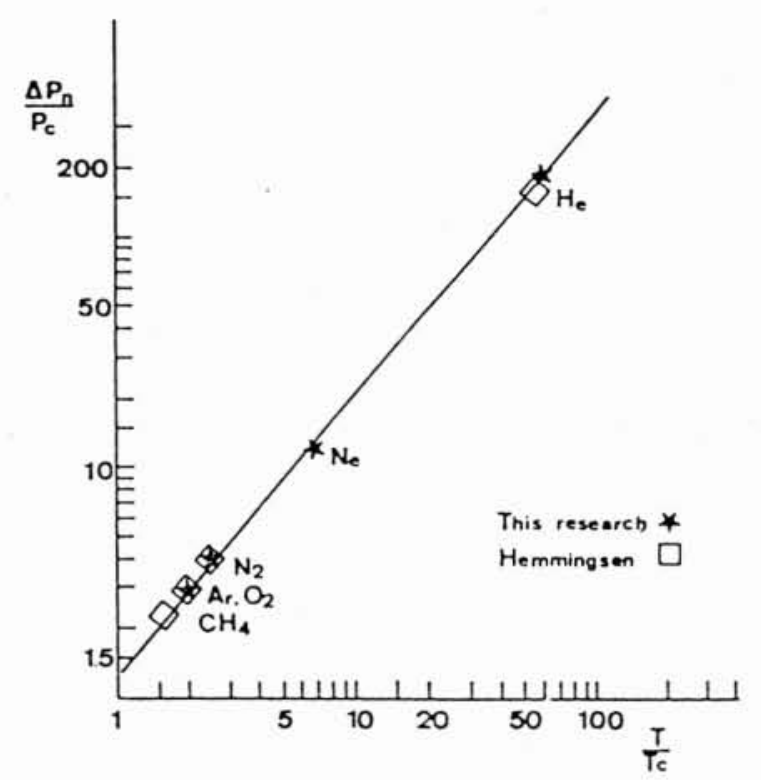

2. Comparaison des mesures de surpression de nucléation homogène avec la corrélation (3) [8].

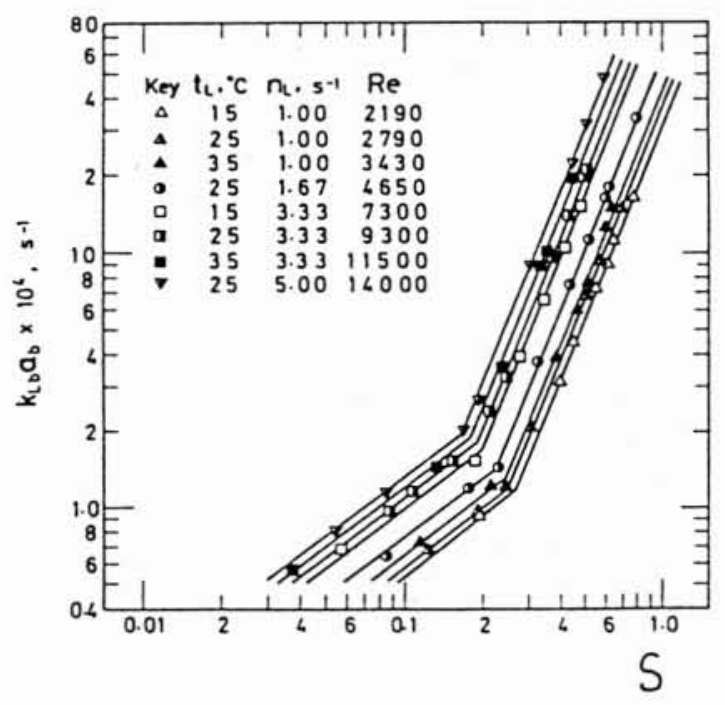

4. Régimes de bullage de Hikita et Konishi [13] en fonction de la sursaturation.

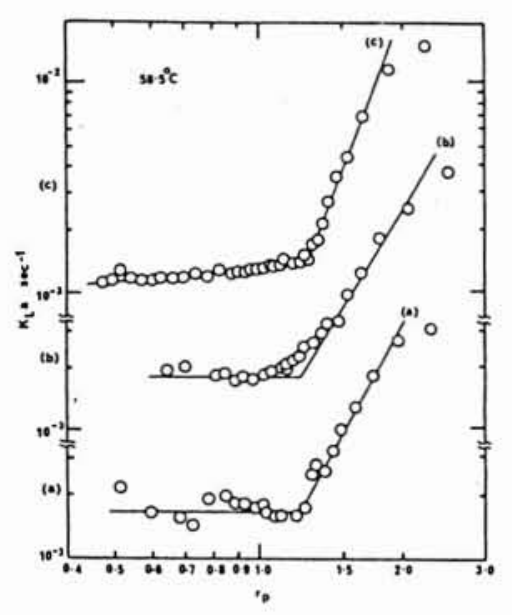

3. Limite entre la désorption tranquille et la désorption nucléée en fonction du rapport de pression $r_{p}$ [32].

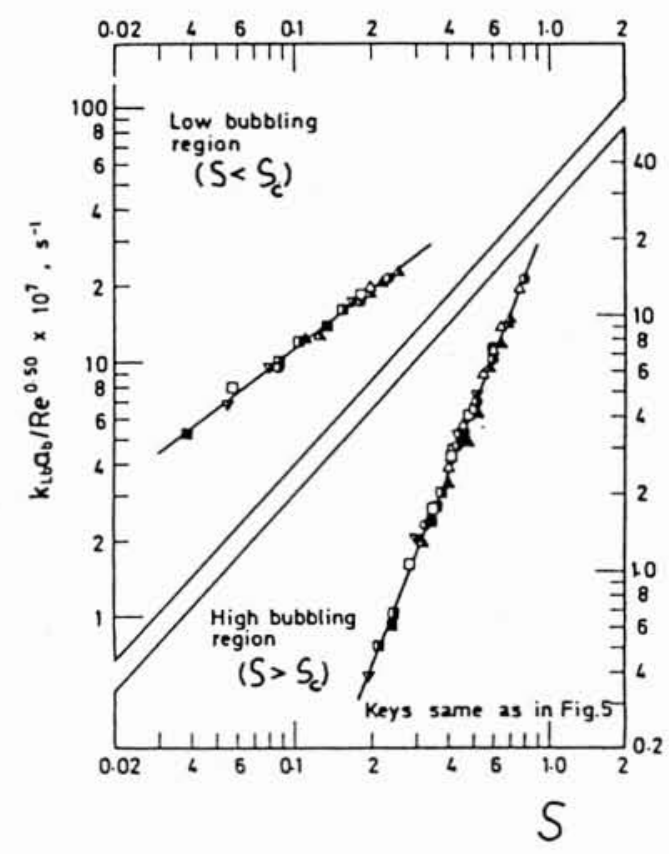

5. Comparaison de la corrélation (5) avec les résultats expérimentaux [13].
Bien que cette relation fournisse souvent des ordres de grandeur raisonnables, tous les problèmes ne sont pas résolus.

- le rayon $R_{0}$ apparaît assez souvent comme un coefficient d'ignorance qui dépend de la forme de l'anfractuosité considérée et de la nature des fluides (en particulier par la notion d'angle de contact). De ce fait, la densité effective des sites de nucléation dépend de la nature du liquide et de la pression $[6,7]$.

- il est à peu près certain à l'heure actuelle que c'est la possibilité pour les anfractuosités d'emprisonner du gaz, qui en fait des sites de nucléation. Les preuves en sont la disparition de la nucléation hétérogène lors de la mise en œuvre de très hautes pressions [8] (dissolution des occlusions gazeuses) ou l'accroissement de la surchauffe nécessaire en vaporisation lorsque le vide est effectué dans le récipient préalablement à son remplissage [3].

- la relation (4) n'est qu'une description a posteriori dans la mesure où les rayons $R_{0}$ sont précisément mesurés par désorption. 


\section{Travaux relatifs à la désorption nucléée en génie chimique}

En fait, ces travaux sont rares et dispersés et il est nécessaire de les citer individuellement.

\subsection{Approches empiriques}

\subsubsection{La notion de délai de nucléation}

Friedel et Molter [9] se sont intéressés au dimensionnement des orifices de dépressurisation des réservoirs de stockage sous pression. Il convient de connaitre le débit de gaz à évacuer et donc la cinétique de désorption du gaz dissous. Lors de leur étude expérimentale du phénomène, les auteurs ont mesuré le " délai de nucléation " comme le temps au bout duquel la nucléation commence de manière visible à partir de l'ouverture de l'orifice de détente. Pour un récipient de verre de 1,7 litre et des solutions de CMC et de polyethylèneglycol saturées en $\mathrm{CO}_{2}$ sous 5 à 15 bars, ce délai vaut entre 75 et $900 \mathrm{~ms}$. Il dépend du degré d'ouverture de l'orifice de dépressurisation et du remplissage du récipient mais semble indépendant de la pression initiale et de la viscosité de la solution. Les auteurs rassemblent leurs résultats par une corrélation empirique sans aucun degré de généralité.

\subsubsection{Limites des régimes de désorption et flux correspondants}

Deux groupes d'auteurs ont étudié la désorption de $\mathrm{CO}_{2}$ d'eau saturée par diminution de la pression statique.

Thuy et Weiland [28] et Weiland et coll. [32] utilisent deux contacteurs gaz-liquide de laboratoire : une cellule agitée à interface plan et une colonne de sphères. La transition entre la désorption tranquille et la désorption nucléée se traduit dans ce type d'appareils par une cassure dans la courbe donnant le coefficient volumétrique de désorption $k_{L} a$ en fonction du rapport de pression $r_{p}$.

$$
k_{L} a V=\frac{F}{C_{L}-C_{L}^{*}} \quad \text { et } \quad r_{p}=\frac{H e C_{L}+p_{\mathrm{H}_{2} \mathrm{O}}}{p_{T}}
$$

La principale conclusion des auteurs est que la désorption cesse d'être tranquille, dès que la pression partielle du gaz dissous est supérieure à la pression totale ambiante. La figure 3 donne un exemple d'un tel profil. Il est à noter qu'il s'agit de la pression partielle du gaz dissous et non de la pression totale qui serait en équilibre avec le liquide; la pression de vapeur saturante du solvant ne joue semble-t-il aucun rôle. Remarquons que la nucléation est observée à des sursaturations qui n'ont rien à voir avec les sursaturations de nucléation homogène (ordre de grandeur: moins de deux bars).

Hikita et coll. [13-14] ont étudié dans un premier temps la désorption de $\mathrm{CO}_{2}$ à partir de solutions salines sursaturées dans un réacteur agité à interface plan, le ciel gazeux au-dessus du liquide pouvant être, soit du $\mathrm{CO}_{2}$, soit de l'azote (domaine de pression 1 à 6 bars). Les auteurs se placent dans des conditions opératoires telles, qu'il y a toujours désorption nucléée; ils mesurent le débit total de désorption qui se compose d'une part de la désorption "tranquille " à la surface (ce flux dépend de la composition du ciel gazeux), d'autre part de la désorption nucléée (indépendante de la composition du ciel gazeux). A partir des mesures effectuées avec les deux compositions du ciel gazeux indiquées, il est possible de séparer les deux contributions :

- la désorption " tranquille " à travers l'interface plan est accélérée d'un facteur $\phi$ par les bulles qui s'échappent;

- on distingue deux comportements de bullage différents suivant la sursaturation du liquide; aux petites valeurs de la sursaturation relative $S$ (régime de bullage faible), le coefficient volumique de désorption nucléée $\left(k_{L} a\right)_{b}$ est proportionnel à la racine carrée de la vitesse d'agitation; aux grandes valeurs de $S$ (régime de bullage fort), la dépendance est plus forte, comme le montre la figure 4. Les auteurs proposent les corrélations empiriques suivantes pour représenter ces résultats :

$$
\begin{gathered}
S \leqslant S_{c} \quad\left(k_{L} a\right)_{b}=6,77 \cdot 10^{-6} f \cdot \operatorname{Re}_{A}^{0,50} S^{0,78} \\
S \geqslant S_{c} \quad\left(k_{L} a\right)_{b}=2,45 \cdot 10^{-6} \operatorname{Re}_{A}^{0,93} S^{2,5}
\end{gathered}
$$

avec :

$$
\begin{gathered}
\operatorname{Re}_{A}=\frac{\mathrm{n}_{\mathrm{A}} \mathrm{d}_{A}^{2}}{v_{L}} \quad S=\frac{C_{L}-C_{L}^{*}}{C_{L}^{*}} \\
S_{c}=1,81 \operatorname{Re}_{A}^{-0,25} \quad \log f=8,36 \cdot 10^{-5.1}
\end{gathered}
$$

Les résultats expérimentaux obtenus par les auteurs sont très bien représentés par ces corrélations, comme le montre la figure 5 .

La présence d'un sel dissous dans l'eau a un effet double : d'une part, la solubilité est affectée (effet de sel classique); d'autre part, le coefficient volumique de désorption nucléée est augmenté. D'après Hikita et Konishi [14], cet effet peut être représenté globalement par un même coefficient $f$, en régime de bullage faible ou fort, qui, de plus, semble être fonction uniquement de la force ionique $I$ de la solution.

Le facteur d'accélération $\phi$ du transfert à l'interface plan dû au bullage est tel que :

$\mathrm{S} \leqslant S_{c} \phi=1 \quad S \geqslant S_{c} \log \phi=522\left(S-S_{c}\right) \operatorname{Re}_{\bar{A}}^{-0.81}$

Les mêmes auteurs [12] ont réussi, avec ces corrélations, à modéliser la désorption nucléée de $\mathrm{CO}_{2}$ due à l'absorption simultanée de $\mathrm{SO}_{2}$ dans une solution d'hydrogénocarbonates et ce, dans le même montage expérimental.

$$
\begin{aligned}
\mathrm{SO}_{2}+\mathrm{HCO}_{3}^{-} & \rightarrow \mathrm{CO}_{2}+\mathrm{HSO}_{3}^{-} \text {(a) } \\
\mathrm{HCO}_{3}^{-}+\mathrm{HSO}_{3}^{-} & \rightarrow \mathrm{CO}_{2}+\mathrm{SO}_{3}^{-}+\mathrm{H}_{2} \mathrm{O} \\
\mathrm{SO}_{2}+\mathrm{SO}_{3}^{-}+\mathrm{H}_{2} \mathrm{O} & \rightarrow 2 \mathrm{HSO}_{3}^{-} \quad \text { (c) } \\
\mathrm{SO}_{2}+\mathrm{H}_{2} \mathrm{O} & \rightarrow \mathrm{HSO}_{3}^{-}+\mathrm{H}^{+} \quad \text { (d) } \\
\mathrm{H}^{+}+\mathrm{HCO}_{3}^{-} & \rightarrow \mathrm{CO}_{2}+\mathrm{H}_{2} \mathrm{O} \text { (e) } \\
\mathrm{H}^{+}+\mathrm{SO}_{3}^{-} & \rightarrow \mathrm{HSO}_{3}^{-} \text {(f) }
\end{aligned}
$$

Ce système obéit au schéma réactionnel relativement complexe ci-dessus, la cinétique de l'ensemble étant régie par la réaction (b) qui reste néanmoins de type rapide. 
Bien que les relations (5) et (6) ne possèdent aucun degré de généralité, dans la mesure ou l'utilisation du nombre de Reynolds pour corréler les résultats n'est pas justifiée expérimentalement, ce travail est un bon exemple des techniques de modélisation utilisées en Génie des procédés.

\subsection{Description de la désorption nucléée par la méthode des bilans de population}

Cette méthode ne vise pas à expliquer le phénomène, mais à le décrire à l'aide d'un formalisme mathématique, incluant des paramètres déterminables expérimentalement, qui permette les calculs. Pasiuk-Bronikowska et Rudzinski [21, 22] étudient la désorption d'azote produit par la réaction d'acide sulfamique avec des ions nitrites dans un réacteur agité :

$$
\begin{aligned}
\mathrm{H}_{2} \mathrm{NSO}_{3}^{-}+\mathrm{H}^{+}+\mathrm{NO}_{2}^{-} & \rightarrow \mathrm{N}_{2}^{\uparrow}+\mathrm{H}^{+}+\mathrm{SO}_{4}^{--} \\
& +\mathrm{H}_{2} \mathrm{O}
\end{aligned}
$$

Ces auteurs décrivent la désorption en utilisant la méthode des bilans de population sur des classes de bulles de taille $L$ donnée. Ils partent du bilan suivant de gaz dissous qui décrit la sursaturation $s=\left(C_{L}-C^{*} L\right)$ d liquide :

$$
\frac{d s}{d t}=r-\frac{F}{V}
$$

$F$ est le flux total de gaz désorbé à la fois par désorption " tranquille " (flux $F_{q}$ ) à travers la limite supérieure de l'émulsion gaz-liquide et par désorption nucléée (flux $F_{b}$ ), $V$ le volume de liquide dans le réacteur et $r$ la vitesse de réaction. Si $N_{q}$ et $N_{b}$ sont respectivement les densités de flux de désorption à la surface de l'émulsion et sur une bulle de diamètre $L$, il vient :

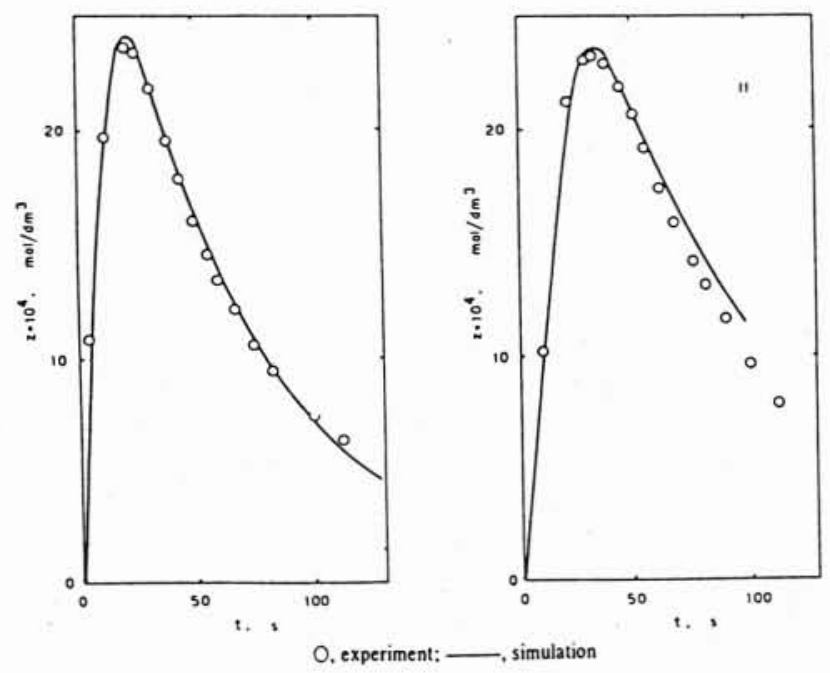

6. Représentation de sursaturations observées à l'aide de la méthode des bilans de population [21,22].
$\frac{F}{V}=N_{q}(s, t) a_{s}+\int_{0}^{L_{\max }} N_{b}(s, L, t) \pi L^{2} \psi(L, t) d L$

$\psi$ est la distribution en nombre des tailles de bulles, solution d'un bilan de population basé sur les hypothèses suivantes :

- les bulles sont supposées sphériques et rigides; on néglige les phénomènes de coalescence et de rupture, - les alimentations et les sorties de la classe $(L ; L+$ $d L$ ) sont constituées par la croissance des bulles et par la sortie du volume réactionnel. Cela se traduit par l'équation aux dérivées partielles suivantes:

$$
\begin{gathered}
\frac{\partial}{\partial t}[V(t) \Psi(L, t)]+\frac{\partial}{\partial L}[G(L, t) V(L, t) \Psi(L, t)] \\
=-f_{b}(L, t) V(L, t) \Psi(L, t) \\
\Psi(L, 0)=0 \quad \Psi(0, t)=\frac{J(t)}{G(0, t)} \\
G(L, t)=\frac{d L}{d t}
\end{gathered}
$$

$G$ est la vitesse de croissance et $f_{b}$ caractérise le flux de sortie par la surface de l'émulsion.

Pour utiliser ces équations, il est nécessaire de disposer d'équations constitutives qui permettent d'estimer $N_{q}, N_{b}, G$ (directement relié à $N_{b}$ ), $f_{b}$ et $J(t)$. On postule généralement la forme de ces relations et les paramètres restant sont déterminés par accord avec l'expérience. Les auteurs postulent les relations ci-après que nous ne justifierons pas:

$$
\begin{aligned}
& N_{b} \cong B_{1} \sqrt{s} \quad G \cong\left(\frac{2 M_{G}}{\rho G}\right) N_{b} \\
& J \cong B_{2} s f_{b}(L, t) \cong B_{3} L
\end{aligned}
$$

Les paramètres $B_{1}, B_{2}$ et $B_{3}$ peuvent être déduits de l'expérience. Les figures 6 issues des travaux de PasiukBronikowska et Rudzinski [22] montrent l'accord entre le modèle optimisé et les résultats expérimentaux relatifs aussi bien au débit de désorption qu'à la sursaturation. Cette technique est une description a posteriori de résultats expérimentaux; elle permet cependant de décrire l'évolution de la distribution de tailles de bulles et l'aire interfaciale et ces travaux sont généralement effectués dans l'espoir d'obtenir des modèles d'extrapolation.

\subsection{La désorption nucléée en génie électrochi- mique}

Il est fréquent de rencontrer des réactions électrochimiques qui conduisent à la production de gaz aux électrodes; la production de gaz peut être la réaction souhaitée (électrolyse), ou au contraire une réaction parasite. Les mécanismes de désorption sont actuellement loin d'être éclaircis.

Dapkus [5] a utilisé une électrode parfaitement lisse (mercure); son étude très soigneuse de la désorption d'hydrogène produit par électrolyse d'une solution 
aqueuse d'acide sulfurique montre que la désorption a lieu par nucléation homogène à faible distance de l'électrode à des sursaturations de l'ordre de $1 \mathrm{MPa}$, alors que la théorie classique de la nucléation homogène donnerait des valeurs environ 150 fois plus élevées et que la corrélation de Finkelstein et Tamir conduirait à des valeurs de l'ordre de $23 \mathrm{MPa}$.

D'autres auteurs ont mesuré des sursaturations en oxygène de l'ordre de $10 \mathrm{MPa}$ au voisinage d'électrodes, ce qui serait compatible avec la limite de nucléation homogène de Finkelstein et Tamir (environ $14 \mathrm{MPa}$ ). L'étude systématique de ces phénomènes de sursaturation au voisinage des électrodes reste à faire et nécessitera, à notre avis, une étude expérimentale fine des bulles et microbulles au voisinage immédiat de l'électrode pendant l'électrolyse.

\section{Conclusions}

Alors que la désorption nucléée intervient dans de nombreux domaines de l'industrie chimique, biochimique et alimentaire, les études la concernant sont assez rares. Il est probable que la théorie classique de la nucléation homogène ne s'applique pas au cas de la désorption d'un gaz à partir d'une solution sursaturée, encore qu'elle semble décrire qualitativement au moins les résultats obtenus par Attar [2] en liquéfaction de charbon; récemment, Finkelstin et Tamir [8] ont indiqué une formule empirique donnant la limite de nucléation homogène dans l'eau et ont proposé une modélisation basée sur la thermodynamique des phénomènes irréversibles et qui rend compte de leurs mesures expérimentales. En génie chimique, la désorption nucléée a presque toujours lieu par nucléation hétérogène et à des sursaturations beaucoup plus faibles que celles conduisant à la nucléation homogène. Comme le nombre et l'efficacité des sites de nucléation ne sont en général pas connus, les types de démarches suivantes ont été proposés pour modéliser les procédés en l'absence de connaissance fondamentale de ce phénomène :

- des démarches purement empiriques qui considèrent le réacteur comme une boîte noire et caractérisent la désorption soit par un délai de nucléation, soit par un coefficient volumique de désorption nucléée, les deux étant ensuite corrélés de façon aveugle en fonction des conditions opératoires;

- la méthode des bilans de population : cette démarche, scientifiquement plus satisfaisante mais tout aussi empirique, n'en reste pas moins limitée à une description a posteriori de phénomènes observés, tant qu'on ne dispose pas de méthode de prédiction du taux de nucléation dans les conditions opératoires réelles de l'industrie.

Dans le domaine particulier de la désorption nucléée sur ou au voisinage d'électrodes, les renseignements dont nous disposons sont trop fragmentaires et quelquefois contradictoires pour permettre une conclusion.

Les voies de recherche suivantes nous semblent intéressantes :

- d'un point de vue fondamental, vérifier la validité du modèle proposé par Finkelstein et Tamir et l'étendre, le cas échéant, à d'autres solvants que l'eau. Que se passe-t-il aux pressions et températures élevées et avec des produits inhabituels (liquides en train de polymériser, métaux, verres ou plastiques fondus)?

- d'un point de vue pratique, il serait utile de mettre au point une technique permettant de caractériser le contenu en germes de nucléation et leur activation dans des liquides quelconques en mouvement. Il faut pouvoir, pour ce faire, assister à la " naissance " des bulles;

- le cas de la désorption aux électrodes nous semble mériter une attention toute particulière au vu de l'importance du sujet et du manque de connaissance actuel car il est clair que la désorption des gaz sur ou au voisinage des électrodes conditionne le fonctionnement de celles-ci.

Adresse des auteurs

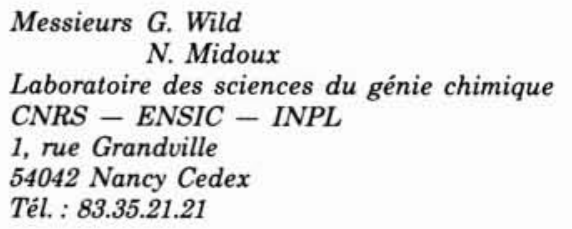




\section{Références}

[1] Aksel'rud G.A., Dubynin A.I. et Gumnitskiy Ya. M. "Heat and mass transfer in chemical reactions accompanied by gas evolution". Heat Transfer: Soviet Research 10 (1978) p. 147-151.

[2] AtTAR A. - "Bubble nucleation in viscous material due to formation by a chemical reaction" AiChE $J 24$ (1978) p. 106-115.

[3] Blander M. - "Bubble nucleation in liquids". Advances Coll. Interf. Sci. 10 (1979) p. 1-32.

[4] Boguslaviski E.A. - "Outgassing kinetics of a supersaturated solution with bubbles growing by diffusion". J. Appld Chem. USSR (Zh. Prikl. Khim.) 58 (1985) p. 1388-1391.

[5] DAPKUS K.V. - "Homogeneous nucleation of electrolytically evolved gasses at an idal electrode" $\mathrm{Ph} \mathrm{D}$ Thesis Carnegie-Mellon University, Pittsburgh, 1985.

[6] Eddington R.I. et Kenning D.B.R. - "The effect of contact angle on bubble nucleation". Int. J. Heat Mass Transfer 22 (1979) p. 1231-1236.

[7] Eddington R.I., Kenning D.B.R. et Korneichev A.I. "Comparison of gas and vapour bubble nucleation on a brass surface in water". Int. J. Heat Mass Transfer 21 (1978) p. 855-862.

[8] Finkelstein Y. et TAMIR A. - "Formation of gas bubbles in supersaturated solutions of gases in water" AIChE J 31 (1985) p. 1409-1419.

[9] FRIEDEL L. et MolTER E. - "Entlöseverzug in gesättigten Gas/Flüssigkeits-Systemen unterschiedlicher Viskosität bei plötzlicher Druckentlastung". Chem.-Ing.Tech. 58 (1986) p. 40-43.

[10] Hemmingsen E.A. - "Effects of surfactants and electrolytes on the nucleation of bubbles in gas-supersaturated solutions". Naturforschung 33a (1978) p. 164-171.

[11] Hemmingsen E.A. - "Cinephotomicrographic observations on intercellular bubble formation in Tetrahymena". $J$. Experimental Zoology 220 (1982) p. 43-48.

[12] Hikita H., AsaI S. et KonISHI Y. - "Absorption of sulfur dioxide into aqueous sodium bicarbonate solution accompanied by bubbling-desorption of carbon dioxide". In "Recent Trends in Chemical Reaction Engineering" (éditeurs B.D. Kulkarni, R.A. Mashelkar et M.M. Sharma) Vol. II p. 30-44, Wiley Eastern Ltd, New Delhi, Inde, 1987.

[13] Hikita H. et Konishi Y. - "Desorption of carbon dioxide from supersatured water in an agitated vessel". AIChE $J$ 30 (1984) p. 945-951.

[14] Hikita H. et Konishi Y. - "Desorption of carbon dioxide from aqueous electrolyte solutions supersaturated with carbon dioxide in an agitated vessel". AIChE $J 31$ (1985) p. $697-699$.

[15] Kafarov V.V., Labutina T.V., Ivanov V.A. et Nikitina A.K. - "Desorption of gases from solutions of chemisorbants". Soviet Chem. Ind. 11-12 (1979) p. 831-833.

[16] Kinsman G.J.M., Hazeldean G.S.F. et Davies M.W. "Physicochemical factors affecting the vaccum deoxidation of steels". J. Iron Steel Inst. (1969) p. 1463-1469.

[17] LenglacÉ C. - "Transferts de matière dans un bioréac- teur" thèse de doctorat, INPL Nancy, 1986.

[18] Mahajanl V.V. et Danckwerts P.V. - "The stripping of $\mathrm{CO}_{2}$ form amine-promoted potash solutions at $100^{\circ} \mathrm{C} "$. Chem. Eng. Sci. 38 (1983) p. 321-327.

[19] Mahajani V.V. et Danckwerts P.V. - "Desorption of $\mathrm{CO}_{2}$ by steam bubbles from amine promoted potash solutions". Chem. Eng. Sci. 38 (1983) p. 499.

[20] Marc I. - "Modélisation de la fermentation de la bière dans un réacteur cylindro-conique: cinétiques d'assimila. tion de sucres et de floculation de levures, de production de $\mathrm{CO}_{2}$ et de métabolites". Thèse de docteur-ingénieur, INPL Nancy, 1982.

[21] PASIUK-BRonikowsKa W. et RUdZINSKI K.J. - "Mathematical model of bubble gas desorption from liquids". Chem. Eng. Sci. 35 (1980) p. 512-518.

[22] PAsiuk-Bronikowska W. et RUdzinski K.J. - "Gas desorption from liquids". Chem. Eng. Sci. 36 (1981) p. 1153-1159.

[23] Роновескі R. et Kucharski E. - "Radanie kinetyki desorpcji $\mathrm{CO}_{2}$ roztworu wodnego weglanow potasowych". Inz. Chem. i Proces. 4 (1983) p. 69-84.

[24] Savage D.W., Astarita G. et Joshi S. - "Chemical absorption and desorption of carbon dioxide from hot carbonate solutions". Chem. Eng. Sci. 35 (1980) p. 1513-1522.

[25] Shah Y.T., Juvekar V.A. et Sharma M.M. - "Criteria for supersaturation during simultaneous absorption-desorption". Chem. Eng. Sci. 31 (1976) p. 671-680.

[26] Shah Y.T., Pangarkar V.G. et Sharma M.M. - Criteria for supersaturation in gas-liquid reactions involving a volatile product". Chem. Eng. Sci. 29 (1974) p. 1601-1612.

[27] Shah Y.T. et Sharma M.M. - "Desorption with or without chemical reaction". Trans. Instn Chem. Engrs 54 (1976) p. $1-41$.

[28] ThuY L.T. et WEILAND R.H. - "Mechanisms of gas desorption from aqueous solution". Ind. Eng. Chem. Fundam. 15 (1976) p. 286-293.

[29] Volmer M. - "Kinetik der Phasenbildung" Theodor Steinkopff Verlag, Dresde, Allemagne, 1939.

[30] Volpicelli G., Caprio V., Santoro L. et Ciambelli P. "Neutralization of acid waters containing aluminium ions by means of limestone". Chem. Eng. J. 21 (1981) p. 29-38.

[31] Wedershoven H.M.S., de Jonge R.M., Sill.en C.W.M.P. et van STRALEN S.J.D. - "Behaviour of oxygen bubbles during alkaline water electrolysis". Int. J. Heat Mass Transfer 25 (1982) p. 1239-1243.

[32] Weiland R.H., Thuy L.T. et Liveris A.N. - "Transition from bubbling to quiescent desorption of dissolved gases". Ind. Eng. Chem. Fundam. 16 (1977) p. 332-335.

[33] WILD G. - "Begasung von Wasser mit gasbeladenen Druckwasserstrahlen - Einfluß eines in Wasser gelösten Zweitgases auf die Absorption von Blasen" Dissertation Universität Karlsruhe, RFA, 1979.

[34] Pellin J. - "European utilities assess compressed-airstorage schemes". Electrical Rev. 11 juillet 1980. 